\title{
Outcome of Extracorporeal Photopheresis as an Add-On Therapy for Antibody-Mediated Rejection in Lung Transplant Recipients
}

\author{
Alberto Benazzo $^{\text {a }} \quad$ Nina Worel $^{b}$ Stefan Schwarz ${ }^{a} \quad$ Ulrike Just $^{c}$ \\ Anna Nechay ${ }^{a}$ Christoph Lambers $^{a}$ Georg Böhmig ${ }^{d}$ Gottfried Fischer $^{b}$ \\ Daniela Koren $^{\mathrm{b}}$ Gabriela Muraközy $^{\mathrm{a}}$ Robert Knobler ${ }^{\mathrm{c}}$ Walter Klepetko ${ }^{\mathrm{a}}$ \\ Konrad Hoetzenecker ${ }^{a}$ Peter Jaksch ${ }^{a}$ \\ ${ }^{a}$ Division of Thoracic Surgery, Department of Surgery, Medical University of Vienna, Vienna, Austria; ${ }^{b}$ Department \\ of Blood Group Serology and Transfusion Medicine, Medical University of Vienna, Vienna, Austria; 'Department of \\ Dermatology, Medical University of Vienna, Vienna, Austria; ${ }^{\mathrm{d}}$ Division of Nephrology and Dialysis, Department of \\ Internal Medicine III, Medical University of Vienna, Vienna, Austria
}

\section{Keywords \\ Extracorporeal photopheresis · Lung transplantation . \\ Antibody-mediated rejection}

\section{Abstract}

Introduction: The diagnosis and treatment of antibody-mediated rejection (AMR) after lung transplantation has recently gained recognition within the transplant community. Extracorporeal photopheresis (ECP), currently used to treat chronic lung allograft dysfunction, modulates various pathways of the immune system known to be involved in AMR. We hypothesize that adding ECP to established AMR treatments could prevent the rebound of donor-specific antibodies (DSA). Objectives: This study aimed to analyze the role of ECP as an add-on therapy to prevent the rebound of DSA. Methods: Lung transplant recipients who received ECP as an add-on therapy for pulmonary AMR between January 2010 and January 2019 were included in this single-center retrospective analysis. Baseline demographics of the patients, as well as their immunological characteristics and long-term transplant outcomes, were analyzed. Results: A total of 41 patients developed clinical AMR during the study period. Sixteen patients received ECP as an add-on therapy after
\end{abstract}

first-line AMR treatment. Among the 16 patients, 2 (13\%) had pretransplant DSA, both against human leukocyte antigen (HLA) class I (B38, B13, and C06). Fifteen patients (94\%) developed de novo DSA (dnDSA), i.e., 10 (63\%) against class I and $14(88 \%)$ against class II. The median time to dnDSA after lung transplantation was 361 days (range 25-2,548). According to the most recent International Society of Heart and Lung Transplantation (ISHLT) consensus report, 2 (13\%) patients had definite clinical AMR, 6 (38\%) had probable AMR, and 7 (44\%) had possible AMR. The median mean fluorescence intensity (MFI) of dnDSA at the time of clinical diagnosis was 4,220 (range 1,319-10,552) for anti-HLA class I and 10,953 (range 1,969-27,501) for anti-HLA class II antibodies. ECP was performed for a median of 14 cycles (range 1-64). MFI values of dnDSA against HLA classes I and II were significantly reduced over the treatment period (for anti-class I: 752; range 70-2,066; for anti-class II: 5,612; range 1,689$21,858)$. The 1 -year survival rate was $55 \%$. No adverse events related to ECP were reported in any of the patients. Conclusions: ECP is associated with a reduction of $\mathrm{dnDSA}$ in lung transplant recipients affected by AMR. Prospective studies are warranted to confirm the beneficial effects of ECP in the setting of AMR. 


\section{Introduction}

Recently antibody-mediated rejection (AMR) has increasingly gained recognition within the lung transplant (LuTx) community due to its poor outcomes [1]. In contrast to other organs, diagnostic criteria for AMR after LuTx were not uniformly defined until 2016, when a consensus report of ISHLT defined 2 forms of pulmonary AMR [1]. According to the report, based on the presence of allograft dysfunction, AMR could be differentiated in clinical or subclinical rejection. In parallel, the following 4 main diagnostic criteria have been identified: measurable allograft dysfunction, circulating donor-specific antibodies (DSA), positive C4d staining, and lung injury histology [1]. Treatment of AMR remains controversial. Current evidence is based on case reports or small singlecenter series, and outcomes remain poor [2-10]. The most used therapies are aimed either at depletion of antibodies or suppression of B and plasma cells. However, the treatment choice is typically the result of the physician's preference and the disease course. In our institution, extracorporeal photopheresis (ECP) was recently introduced as an add-on therapy with the ultimate purpose of preventing the rebound of DSA. To the best of our knowledge, this retrospective analysis is the first report of ECP use after pulmonary AMR.

\section{Materials and Methods}

Between January 2010 and December 2019, one thousand eleven patients received primary lung transplantation at the Medical University of Vienna. Our database was reviewed for patients who were diagnosed with clinical AMR and received ECP as an adjunct therapy. Demographics of the patients, as well as humoral specific data and survival data, were analyzed.

\section{Clinical Protocol}

All patients received induction therapy with alemtuzumab (a single bolus of $30 \mathrm{mg}$ upon arrival at the intensive care unit). The maintenance immunosuppression regimen included tacrolimus, mycophenolate mofetil (MMF), and steroids. Tacrolimus was started immediately after transplantation with target blood levels of $8-10 \mathrm{ng} / \mathrm{mL}$ in the first year posttransplantation, $6-8 \mathrm{ng} / \mathrm{mL}$ during the second year, and $5 \mathrm{ng} / \mathrm{ml}$ afterward. MMF was started 1 year after transplantation with a daily dose of 1-3 g. In patients who experienced a decrease in their glomerular filtration rate $(<60$ $\mathrm{mL} / \mathrm{min}$ ), everolimus was used to reduce the dose of calcineurin inhibitors. Perioperative infectious prophylaxis consisted of broad-spectrum antibiotics adapted to pretransplant colonization. All patients received lifelong pneumocystis prophylaxis with trimethoprim-sulfamethoxazole. Inhalation with amphotericin B was provided for 2-3 months. In patients with cystic fibrosis, inhalation with colistin or tobramycin was performed for a maximum of 12 months posttransplantation. Cytomegalovirus (CMV) prophylaxis included CMV hyperimmunoglobulines and valganciclovir for a minimum of 3 months. In high-risk patients (donor: CMV IgG positive, recipient: CMV IgG negative) a 12-month prophylaxis was performed.
The standard posttransplantation care included a surveillance bronchoscopy with a transbronchial biopsy and bronchoalveolar lavage, spirometry with body plethysmography at 2 weeks and 1, 2, 3, 6, and 12 months after transplantation, computed tomography (CT) scan (each year), and regular blood examination. De novo DSA (dnDSA) are routinely tested after transplantation at 2 weeks and 1, 2, 3, 6, 9, 12, 18, and 24 months after transplantation and in cases of lung function deterioration. Additional diagnostic transbronchial biopsy, bronchoalveolar lavage, and CT scans were performed in cases of lung function deterioration. Biopsies were classified according to ISHLT criteria $[11,12]$. AMR was classified according to the most recent ISHLT consensus report [1].

\section{Acute Rejection Treatment}

Acute cellular rejection (grade A2) and lymphocytic bronchiolitis (B2 or higher) were primarily treated with steroids (500$1,000 \mathrm{mg} /$ day) for 3 days, and in cases of nonresponse to steroids anti-thymocyte globulin (ATG) $(2 \mathrm{mg} / \mathrm{kg}$ ) was administered for 5 days. In our center AMR is treated with immunoadsorption (IA) as first-line treatment in the majority of cases. For IA patients plasma was separated by centrifugation (COBE Spectra ${ }^{\circledR}$ or Spectra OPTIA $^{\circledR}$ Apheresis System; Terumo BCT) under combined regional and systemic anticoagulation with both citrate and heparin. During each IA session, 2.5 times the patient's plasma volume was processed. By use of the ADAsorb ${ }^{\circledR}$ device (Medicap GmbH, Ulrichstein, Germany) plasma was pumped alternatively through a regenerative GAM146 peptide-coated twin column system (Globaffin ${ }^{\circledR}$; Fresenius Medical Care, Bad Homburg, Germany). Since 2015, all patients with AMR received ECP after the first-line treatment as add-on therapy. In the case of the use of intravenous immunoglobulins, Privigen ${ }^{\circledR}$ (CSL Behring, Germany) was administered with a daily dose of $1-2 \mathrm{~g} / \mathrm{kg}$. The treatment duration was 3-5 days depending on the clinical course. Since 2016, all patients with AMR received ECP as an add-on treatment. ECP was performed using the THERAKOS ${ }^{\mathrm{TM}}$ UVAR $\mathrm{XTS}^{\mathrm{TM}}$ and later the THERAKOSTM CELLEX ${ }^{\text {TM }}$ photopheresis system (Therakos UK Ltd., a Mallinckrodt Pharmaceuticals company), which is a closed-loop sterile system. The procedure has been described in detail elsewhere [13]. During ECP, peripheral blood mononuclear cells are separated from the whole blood in a Latham centrifuge (Latham International, Chesterton, UK) at 2,700 rpm. The collected cells (buffy coat bag) are treated with 8-methoxypsoralen solution (Uvadex; Therakos) and exposed extracorporeally to ultraviolet A light $\left(1-2 \mathrm{~J} / \mathrm{m}^{2}\right)$ before reinfusion into the patient. During each treatment, 4-6 collection cycles were performed or 1,500 $\mathrm{mL}$ blood was processed, depending on the patient's hematocrit level. ECP was started within 1 week after first-line treatment. Initially a 2-day treatment cycle was performed once a week. In case of stabilization of lung function and improvement of the clinical situation, after 3 months a 2-day treatment ECP cycle was performed once a month for a minimum of 6 months. Stabilization or improvement of lung function was defined as a $10 \%$ improvement in $\mathrm{FEV}_{1}$ from the start of ECP. In patients for whom no lung function was available, weaning from ventilation support was defined as clinical improvement.

\section{Human Leukocyte Antigen Antibody Screening}

Patients were screened using a bead array technique (LABScreen ${ }^{\mathrm{TM}}$ Mixed; OneLambda, Canoga Park, CA, USA); in case of a positive screening result, antibody identification was performed using beads covered with single recombinant antigens (LABScreen ${ }^{\circledR}$ Single Antigen; OneLambda). Reactions with an MFI $>1,000$ were considered positive. 
Table 1. Demographics

\begin{tabular}{ll}
\hline Demographics & Value \\
\hline Female gender & $8(50)$ \\
Age, years & $41(20-63)$ \\
Underlying disease & \\
$\quad$ COPD & $2(12.5)$ \\
Idiopathic fibrosis & $1(6)$ \\
CF & $5(31.3)$ \\
iPAH & $2(12.5)$ \\
Sclerodermia & $1(6.3)$ \\
EAA & $2(12.5)$ \\
Sarcoidosis & $2(12.5)$ \\
PPFE & $1(6.3)$ \\
Previous pregnancy & $3(18.8)$ \\
Prolonged postoperative ECMO & $5(31.3)$ \\
PGD grade at 72 h & \\
0 & $12(75)$ \\
1 & $1(6.3)$ \\
2 & 0 \\
3 & 0 \\
Ungradable & $3(18.8)$ \\
High-risk CMV match & $4(25)$ \\
Higher-grade ACR & $2(12.5)$ \\
Higher-grade LB & $2(12.5)$ \\
CLAD phenotype & \\
Obstructive & $5(31.3)$ \\
Restrictive & $3(18.8)$ \\
Mixed & 0 \\
CLAD grade & \\
0 & $9(56.3)$ \\
1 & $1(6.3)$ \\
2 & 0 \\
3 & $6(37.6)$ \\
4 & 0 \\
Retransplantation & $3(18.8)$ \\
\hline & \\
\hline &
\end{tabular}

The total number of patients was 16 . Values are presented as numbers (\%) or medians (range). COPD, chronic obstructive pulmonary disease; iPAH, idiopathic pulmonary arterial hypertension; CF, cystic fibrosis; PPFE, pleuropulmonary fibroelastosis; EAA, extrinsic allergic alveolitis; ECMO, extracorporeal membrane oxygenation; PGD, primary graft dysfunction; ACR, acute cellular rejection; LB, lymphocytic bronchiolitis.

Statistical Analysis

Categorical variables are reported as percentages and continuous variables as reported as medians (range) or means $( \pm \mathrm{SD}) \cdot \chi^{2}$ tests, Fisher's exact tests, Mann-Whitney U tests, or ANOVA were used to compare variables as applicable. Survival curves were generated with the Kaplan-Meier method and compared using logrank tests. Data were analyzed using SPSS version 26 software and figures were designed with GraphPad Prism 6.

\section{Results}

\section{Demographics}

Detailed demographics are presented in Table 1. Within the study period, 41 (4\%) patients were diagnosed with
Table 2. Humoral characteristics

\begin{tabular}{lc}
\hline Characteristic & Value \\
\hline Pretransplantation DSA & $2(12.5)$ \\
Pretransplantation DSA HLA class I & $2(12.5)$ \\
Pretransplantation DSA HLA class II & 0 \\
dnDSA & $15(94)$ \\
Time to dnDSA, days & $361(25-2,548)$ \\
dnDSA HLA class I & $11(73)$ \\
dnDSA HLA class II & $14(88)$ \\
ISHLT AMR grade & \\
$\quad$ Definite & $2(13)$ \\
$\quad$ Probable & $6(40)$ \\
$\quad$ Possible & $7(44)$ \\
Time to AMR, days & $307(27-2,548)$ \\
Class I MFI at AMR diagnosis, $n$ & $4,220(1,319-10,552)$ \\
Class II MFI at AMR diagnosis, $n$ & $10,953(1,969-27,501)$ \\
First-line treatment & $1(6.3)$ \\
$\quad$ ATG & $10(63)$ \\
$\quad$ Immunoadsorption & $2(12.5)$ \\
Combination of IvIg and IA & $2(12.5)$ \\
Combination of ATG and IA & $1(6.3)$ \\
Combination of ATG and IvIg & $10(5-12)$ \\
Cycles of immunoadsorption, $n$ & $2,119(441-9,298)$ \\
Class I MFI after first-line therapy, $n$ & $13,092(2,032-21,655)$ \\
Class II MFI after first-line therapy, $n$ & $14(1-64)$ \\
Cycles of extracorporeal photopheresis, $n$ & $752(70-2,066)$ \\
Class I MFI after ECP, $n$ & $5,612(1,689-21,858)$ \\
Class II MFI after ECP, $n$ & \\
&
\end{tabular}

Values are presented as numbers (\%) or medians (range). IvIg, intravenous immunoglobulins.

clinical AMR and 16 received ECP as an add-on therapy after first-line AMR treatment. In 2016 the first patient was treated with ECP as adjunct therapy. Afterwards, all of the patients underwent this therapy. Eight patients $(50 \%)$ were female and 3 of them had had pregnancies prior to transplantation. Most of the patients had cystic fibrosis as an underlying diagnosis, and $2(12.5 \%)$ patients were transplanted for sarcoidosis, 2 (12.5\%) for extrinsic allergic alveolitis, and 1 (6.3\%) for scleroderma. In 5 patients, VA-ECMO was prolonged in the postoperative period according to our institutional preventive strategy for primary graft dysfunction (PGD) $[14,15]$. Twelve (75\%) patients had PGD grade 0 at $72 \mathrm{~h}, 1$ patient had PGD grade 1, and 3 patients had ungradable PGD. All of the patients received alemtuzumab as induction therapy.

\section{$D S A$ and $A M R$}

Immunological characteristics, AMR grades and treatment strategies are provided in Table 2. Among the 16 patients, $2(13 \%)$ had pretransplant DSA, both against human leukocyte antigen (HLA) class I (B38, B13, and C06). Fifteen patients (94\%) developed dnDSA, i.e., 11 (73\%) against class I and 14 (88\%) against class II. The 


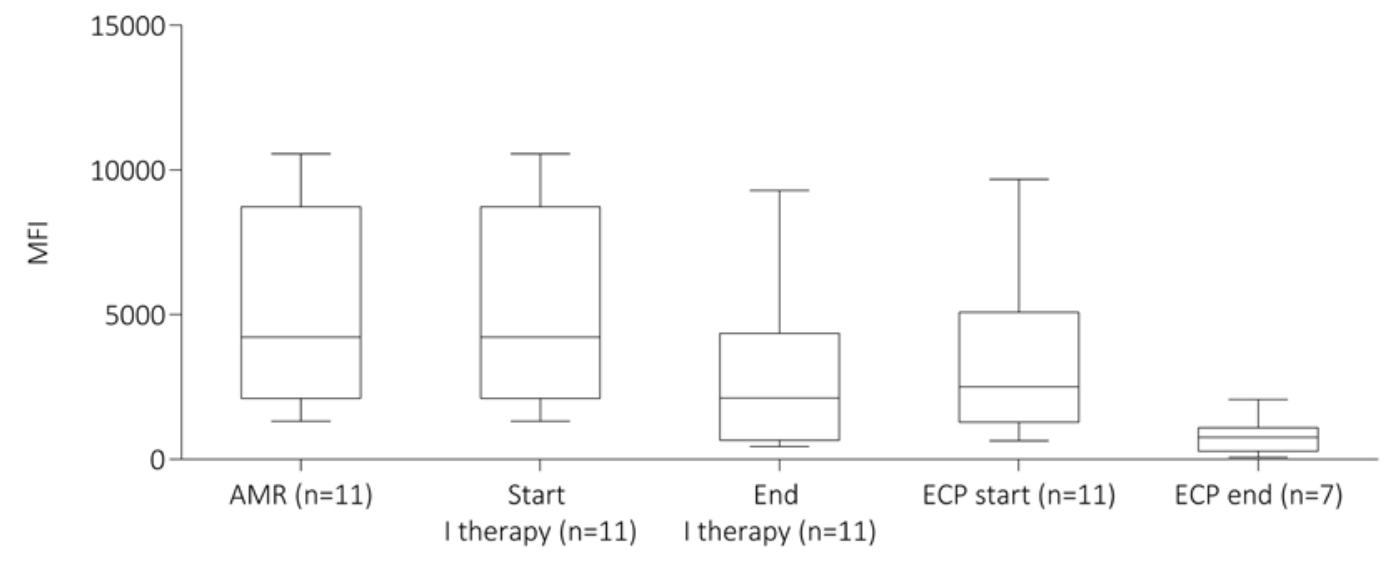

b

HLA Class II

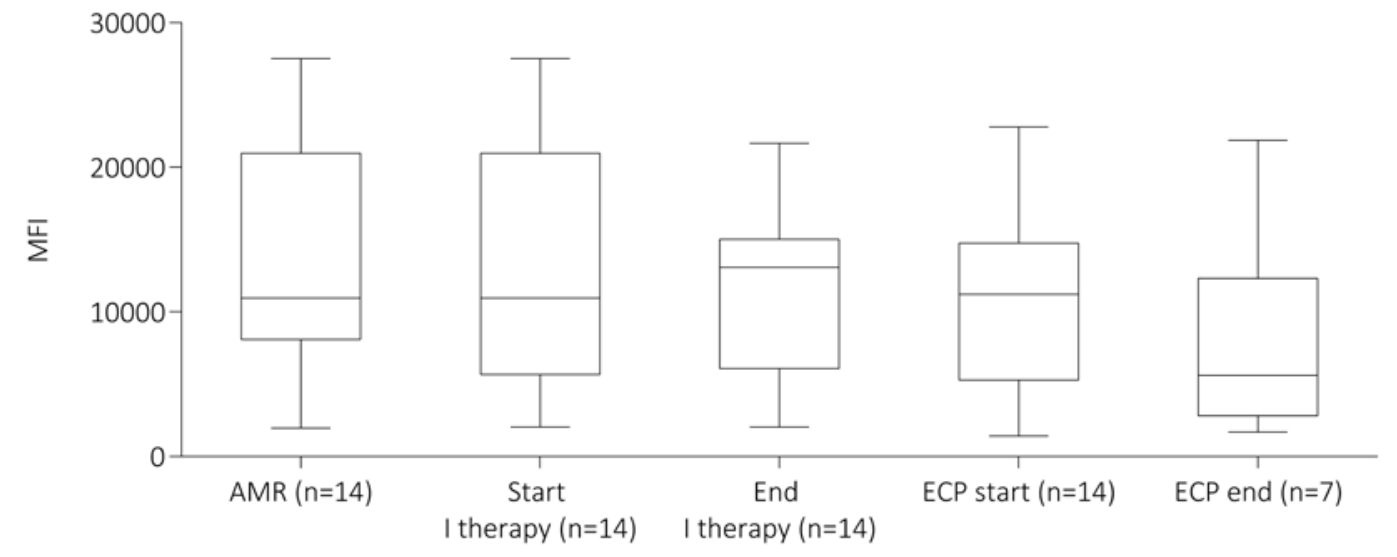

Fig. 1. MFI course of DSA against HLA class I and II measured by Luminex. a MFI of dnDSA against HLA class I was reduced under first-line therapy. However, only after ECP treatment DSA were nearly undetectable. AMR (median: 4,220; range 1,319-10,552), start I therapy (median: 4,200; range 1,300-10,500), end I therapy (median: 2,119; range 411-9,298), ECP start (median: 2,509; range 646-9,675), and ECP end (median: 752; range 70-2,066). b Mea- sured MFI of dnDSA against HLA class II was higher compared to class I and did not decrease after first-line treatment. Nevertheless, ECP led to a reduction and stabilization of MFI. AMR (median: 10,953; range 1,969-27,501), start I therapy (median: 10,976; range 2,032-27,520), end I therapy (median: 13,092; range 2,020-21,655), ECP start (median: 11,206; range 1,407-22,784), and ECP end (median: 5,612; range 1,689-21,858). median time to dnDSA was 361 days (range 25-2,548). In 1 patient no circulating dnDSA were detectable. However, anti-angiotensin-converting enzyme antibodies could be found at the time of clinical deterioration. Based on ISHLT criteria, 2 (13\%) patients had definite clinical AMR, 6 (38\%) had probable AMR, and 7 (44\%) had possible AMR. The median MFI of dnDSA at the time of clinical diagnosis was 4,220 (range 1,319-10,552) for anti-HLA class I and 10,953 (range 1,969-27,501) for anti-
HLA class II. A slight reduction in MFI was observed for dnDSA anti-HLA class I (median: 2,119; range 441$9,298)$ after first-line treatment. The same was not seen for dnDSA anti-HLA class II (median: 13,092; range 2,032-21,655). ECP was performed for a median of 14 cycles (range 1-64). The median MFI of dnDSA against HLA classes I and II was significantly reduced over the treatment period (for anti-class I: 752; range 70-2,066; for anti-class II: 5,612; range 1,689-21,858) (Fig. 1). 


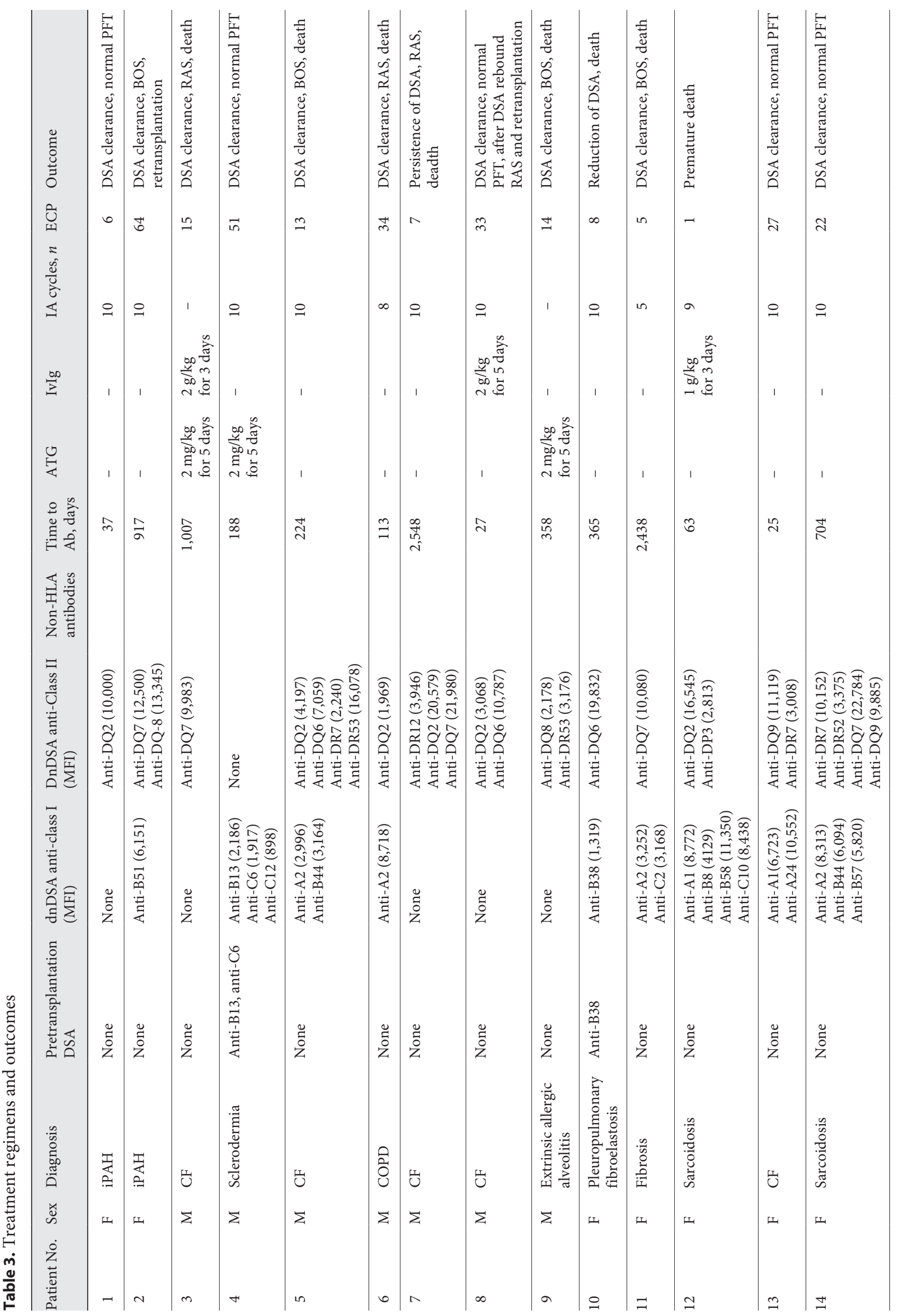


Table 3 shows the treatment regimens used in each patient.

Treatment Regimen 1: IA and ECP

Ten (63\%) patients received IA for DSA depletion (median IA cycles: 10; range 5-10), followed by ECP for a median of 10 cycles (range 5-64). Four of them received less than 10 ECP cycles. All of them, in fact, clinically deteriorated and died of respiratory failure within 6 months of the AMR diagnosis. In 1 patient, 6 cycles of ECP were performed with clearance of DSA and clinical improvement. One further patient underwent 13 ECP cycles. However, he developed chronic lung allograft dysfunction (CLAD) stage 3 with an obstructive phenotype and died of respiratory failure 1 year after AMR diagnosis. The remaining 4 patients received ECP with a median of 34 cycles (range 22-64). In all of them, dnDSA titers could be successfully reduced (MFI anti-class I: 765; range 70-2,066; MFI anti-class II: 4,744; range 1,68921,858).

Treatment Regimen 2: IA plus IVIG and ECP

Two (12.5\%) patients underwent IA, followed by intravenous immunoglobulins due to persistent high dnDSA anti-HLA class II. Despite combined treatment, the MFI of dnDSA did not decrease and ECP was initiated. In 1 of the 2 patients, $33 \mathrm{ECP}$ cycles were performed with a significant reduction of dnDSA MFI (for anti-HLA B7: 5 vs. 3,300; for anti-HLA B8: 0 vs. 4,052; for anti-HLA DQ2: 1,883 vs. 14,087 ; and for anti-HLA DQ6: 8,612 vs. 14,239). After 33 cycles, the patient required hospitalization due to a severe infection, which was followed by a rebound in anti-HLA DQ6 and abrupt clinical deterioration. The acute therapy consisted of a combination of 10 cycles of IA and a single dose of alemtuzumab, which reduced the MFI of dnDSA anti-HLA DQ6. The patient developed restrictive CLAD grade 4 within 60 days after the second AMR episode and was retransplanted within 4 months. The second patient started ECP but dies of respiratory failure after 1 cycle.

Treatment Regimen 3: ATG plus IA and ECP

Two patients presented with respiratory failure and ground-glass opacities at CT scans and required noninvasive positive-pressure ventilation. With clinical suspicion of acute rejection, both received pulse steroids and ATG without improvement. In patient No. 16 , circulating $\operatorname{dnDSA}$ were detectable within a few days (MFI for anti-A1:3,972; for anti-B8: 3,672; for anti-B13: 1,370; for anti-DR17: 2,624; for anti-DR7: 9,671; for anti-DR53: 16,422; for anti-DQ2: 26,459; and for antiDP4: 3,884$)$. In patient No. 4 , tissue DSA were found along with positive histological characteristics for AMR. 


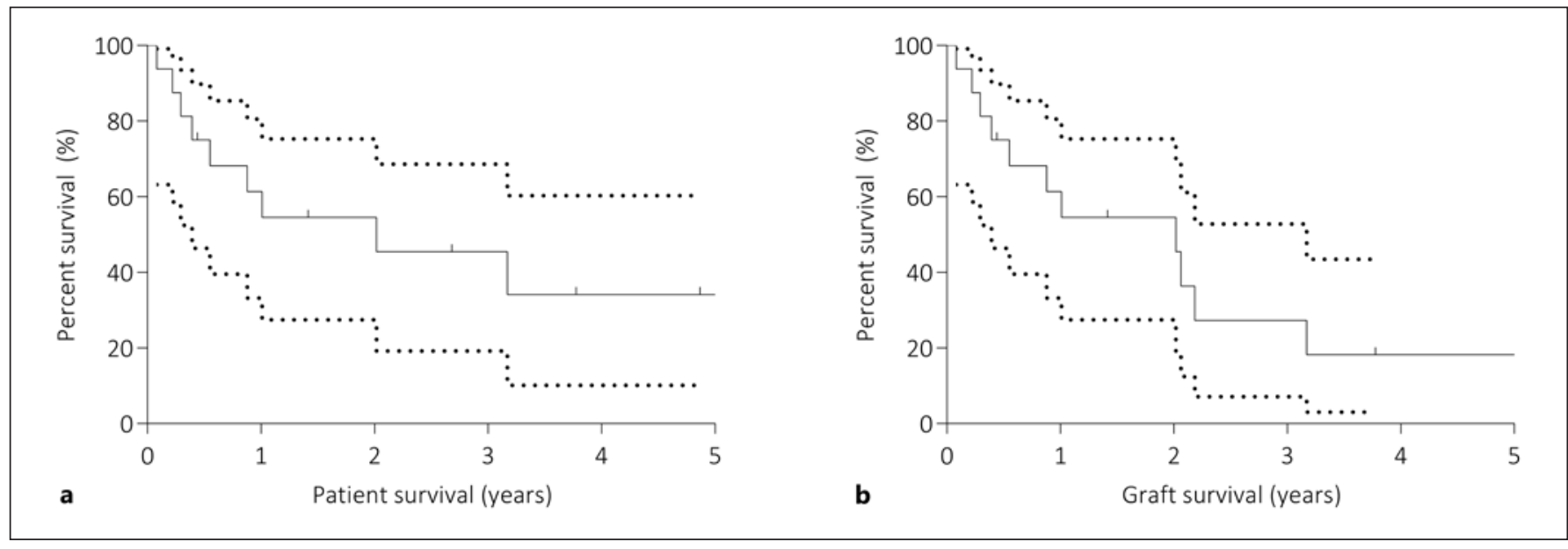

Fig. 2. Kaplan-Meier curve for patient and graft survival. a, b Patient and graft survival after AMR diagnosis. Similar to the international trends, the rate of mortality and graft loss dramatically increased after AMR, with approximately a 50\% mortality rate 2 years after diagnosis. The continuous line represents overall survival, and the dashed line represents $95 \% \mathrm{CI}$.

Both patients underwent 10 and 12 cycles of IA, respectively, after which only partial clearance of dnDSA was observed. After ECP was started, patients could be weaned from noninvasive positive-pressure ventilation and discharged with restored lung function. Three months after the start of ECP, dnDSA were cleared entirely except for DSA anti-HLA DQ2 and lung function was restored.

Treatment Regimen 4: ATG or ATG + IVIG and ECP In patients No. 3 and No. 9 a possible clinical AMR was diagnosed based on clinical deterioration and circulating dnDSA after exclusion of all other possible causes. In both patients the histology was negative. Patient No. 3 received IVIG and ATG consecutively (MFI for antiA26: 1,074; for anti-DQ7: 9,933; and for anti-DQ6: 2,067) while patient No. 9 received only ATG (MFI for antiDQ8: 2,178 and for anti-DR53: 3,176). A partial reduction in MFI was observed in both cases and ECP was initiated. DSA were cleared but both patients developed CLAD stage 3 (1 had bronchiolitis obliterans syndrome and 1 had restrictive allograft syndrome) within 1 year. They died 737 and 321 days after the AMR diagnosis due to respiratory failure.

\section{Long-Term Outcomes}

Eight patients developed CLAD during the follow-up; $5(31 \%)$ had BOS and $3(19 \%)$ had RAS. Three (19\%) patients underwent retransplantation due to end-stage CLAD. Kaplan-Meier survival curves for patient and graft survival are shown in Figure 2. The 1- and 5-year patient survival rates after AMR diagnosis were 54 and $34 \%$, respectively. The $1-$ and 5 -year graft survival rates were 61 and 18\%, respectively.

\section{Discussion/Conclusion}

Although significant improvement has been made in the diagnostic accuracy of AMR after lung transplantation, the treatment options are still limited and longterm outcomes remain poor. According to International Society of Heart and Lung Transplantation (ISHLT) data, in fact, AMR is associated with a $50-70 \%$ mortality rate [1]. Several treatment regimens have been described; however, the published data are based only on small case series. This current study aimed to evaluate ECP as an add-on therapy for AMR. We hypothesized that, after clinical improvement driven by first-line treatment, ECP triggers immune tolerance, thereby improving longterm outcomes. Our retrospective analysis shows that ECP is able to reduce the titers of circulating dnDSA successfully. However, patient and graft survival remains poor.

Donor-specific anti-HLA antibodies (DSA) have been associated with glomerulopathy in kidney transplants and with cardiac allograft vasculopathy in heart transplant recipients $[16,17]$. In lung transplantation, associations between dnDSA and decreased survival, as well as a higher incidence of CLAD, have been previously described $[8,18-21]$. Moreover, poor outcomes have been found in the case of persistence of dnDSA despite antibody-depleting strategies [22]. The generally accepted pathogenic hypothesis of pulmonary AMR is that activated B cells and plasma cells produce antibodies against donor lung antigens after T-cell or dendritic cell activation [1]. The antigen-antibody complex leads to an immune reaction in the lung allografts, resulting in graft injury and dysfunction [1]. Based on this assumption, treatment regimens for AMR aim to 
deplete antibodies or suppress the activation of B and plasma cells. To date, however, it is difficult to identify the best strategy as multiple treatments are usually instituted concomitantly. ECP has only been used in a limited number of patients with AMR after solid-organ transplantation. In a case series of 4 heart transplant recipients with elevated DSA, prophylactic ECP leads to a reduction of circulating antibodies as well as a low incidence of rejection [23]. Similarly, in a pilot study randomizing 23 cardiac recipients to standard maintenance immunosuppression or additive prophylactic ECP, the second group showed less DSA within 6 months after transplantation [24]. In a study investigating the effectiveness of ECP to clear DSA in BOS patients, $19(70 \%)$ patients showed a decrease or stabilization of DSA during the ECP treatment [25]. All patients, however, received concomitantly intravenous immunoglobulins and 18 of them received rituximab [25]. To the best of our knowledge, the present analysis reports the first experience of ECP as an add-on treatment for clinical AMR, showing a clearance or reduction of DSA in most of the patients. ECP may promote tolerance towards donor lung antigens, thereby suppressing plasma cell activation and antibody production. ECP is known to induce T regulatory cells [26] and suppress IL-6 production [27], which in turn could inhibit T-cell-mediated B-cell activation.

Depletion of dnDSA or inactivation of their complement-binding activity seems to be associated with better outcomes. In a study of Witt et al. [9], patients who experienced clearance of dnDSA had better survival compared to those with persistent DSA [9]. Similarly, in a recent study, patients whose DSA lost complementbinding activity were less likely to develop CLAD after AMR resolution [28]. In our study, in 14 patients, dnDSA cases were cleared during ECP treatment; however, 6 of them developed CLAD, and only $26 \%$ of the patients were still alive after 5 years. This is in accordance with the international experience. On the contrary, better outcomes are observed in the field of AMR in kidney recipients, where the use of IA was associated with a survival benefit [29]. A possible hypothesis is that in some cases the injury resulting from the acute phase of AMR is irreversible despite DSA clearance, thereby activating fibroproliferative mechanisms in the allograft. Moreover, concomitant infection of lung recipients may worsen patients' conditions, thereby negatively influencing the outcomes.

The majority of patients $(n=14 ; 88 \%)$ in our cohort had dnDSA against HLA class-II and, more specifically, against $\mathrm{DQ}$ locus. Moreover, 2 patients experienced a rebound of DSA anti-HLA DQ2 and DQ6, respectively, leading to retransplantation in 1 case and death in the second case. This finding is in concordance with the pub- lished experience in solid-organ transplantation [22, 30, 31]. The presence of DSA anti-HLA class II and, in particular, against the DQ locus after kidney transplantation is associated with reduced graft survival [31-33]. In cardiac transplantation, anti-HLA DQ DSA are the predominant subtype found in AMR [34]. Similarly, DQ specificity has been also described in lung transplantation [2]. A possible explanation is that endothelial cells express class II HLA molecules in a proinflammatory environment [35] and, as a consequence, endothelial cells are the starting point of $A M R$.

This study has several limitations. First, its retrospective nature poses a potential bias. Although the single-center approach guarantees a homogenous patient cohort and a consistent granularity of data, variability between different transplant centers is neglected. Besides, we cannot exclude that the decline of dnDSA is attributable to the natural course and physiologic clearance [36]. Due to the low number of patients receiving ECP and the heterogeneity of the first-line treatment strategy, conclusions about the safety of the treatment cannot be drawn and further studies are necessary to confirm our findings. Finally, a control group is missing in the present analysis.

In conclusion, this retrospective analysis reports about the first experience with ECP as a treatment of AMR after lung transplantation. DSA could be cleared in most of the patients and, in approximately $50 \%$ of the patients, lung function could be restored. A multicenter prospective study is needed to investigate the application of ECP in AMR further.

\section{Statement of Ethics}

This study was conducted ethically in accordance with the World Medical Association Declaration of Helsinki and was approved by our institutional ethics commission (EK No. 1139/2020)

\section{Disclosure Statement}

P.J. and N.W. report speakers' fees and research grants from Therakos Mallinckrodt.

\section{Author Contributions}

Alberto Benazzo: data collection and analysis and preparation and finalization of this paper. Stefan Schwarz, Anna Nechay, Ulrike Just, Christoph Lambers, Georg A. Böhmig, Gottfried Fischer, Daniela Koren, Gabriela Muraközy, Walter Klepetko, Konrad Hoetzenecker, and Nina Worel, correction of this paper. Peter Jaksch: data analysis and correction of this paper. Robert Knobler: study design and correction of this paper. 


\section{References}

1 Levine DJ, Glanville AR, Aboyoun C, Belperio J, Benden C, Berry GJ, et al. Antibody-mediated rejection of the lung: A consensus report of the International Society for Heart and Lung Transplantation. J Heart Lung Transplant. 2016 Apr;35(4):397-406.

2 Lobo LJ, Aris RM, Schmitz J, Neuringer IP. Donor-specific antibodies are associated with antibody-mediated rejection, acute cellular rejection, bronchiolitis obliterans syndrome, and cystic fibrosis after lung transplantation. J Heart Lung Transplant. 2013 Jan;32(1): 70-7.

3 Hayes D Jr, DiPaola N, Baker PB, Kirkby S, Phillips AB, Nicol KK. Antibody-mediated rejection in a lung transplant recipient after acute stroke. Transpl Immunol. 2012 Dec; 27(4):171-4.

4 Jackups R Jr, Canter C, Sweet SC, Mohanakumar T, Morris GP. Measurement of donorspecific HLA antibodies following plasma exchange therapy predicts clinical outcome in pediatric heart and lung transplant recipients with antibody-mediated rejection. J Clin Apher. 2013 Aug;28(4):301-8.

5 Baum C, Reichenspurner H, Deuse T. Bortezomib rescue therapy in a patient with recurrent antibody-mediated rejection after lung transplantation. J Heart Lung Transplant. 2013 Dec;32(12):1270-1.

6 Beaty CA, Yang A, George TJ, Illei PB, Shah AS. Unilateral humoral rejection after reoperative single-lung transplant. Ann Thorac Surg. 2013 Apr;95(4):e79-81.

7 Daoud AH, Betensley AD. Diagnosis and treatment of antibody mediated rejection in lung transplantation: a retrospective case series. Transpl Immunol. 2013 Jan;28(1):1-5.

8 Morrell MR, Patterson GA, Trulock EP, Hachem RR. Acute antibody-mediated rejection after lung transplantation. J Heart Lung Transplant. 2009 Jan;28(1):96-100.

9 Witt CA, Gaut JP, Yusen RD, Byers DE, Iuppa JA, Bennett Bain K, et al. Acute antibody-mediated rejection after lung transplantation. J Heart Lung Transplant. 2013 Oct;32(10): 1034-40.

10 Stuckey LJ, Kamoun M, Chan KM. Lung transplantation across donor-specific antihuman leukocyte antigen antibodies: utility of bortezomib therapy in early graft dysfunction. Ann Pharmacother. 2012 Jan;46(1):e2

11 Stewart S, Fishbein MC, Snell GI, Berry GJ, Boehler A, Burke MM, et al. Revision of the 1996 working formulation for the standardization of nomenclature in the diagnosis of lung rejection. J Heart Lung Transplant. 2007 Dec;26(12):1229-42.

12 Berry G, Burke M, Andersen C, Angelini A, Bruneval P, Calabrese F, et al. Pathology of pulmonary antibody-mediated rejection: 2012 update from the Pathology Council of the ISHLT. J Heart Lung Transplant. 2013 Jan;32(1):14-21.

13 Marques MB, Tuncer HH. Photopheresis in solid organ transplant rejection. J Clin Apher. 2006 Apr;21(1):72-7.
14 Hoetzenecker K, Benazzo A, Stork T, Sinn K, Schwarz S, Schweiger T, et al.; Vienna Lung Transplant Group. Bilateral lung transplantation on intraoperative extracorporeal membrane oxygenator: an observational study. J Thorac Cardiovasc Surg. 2019 Nov;S00225223(19)33065-X.

15 Hoetzenecker K, et al. Intraoperative ECMO and the possibility of postoperative prolongation improve survival in bilateral lung transplantation. J Thorac Cardiovasc Surg. 2018 May;155(5):2193-206.e3.

16 Hidalgo LG, Campbell PM, Sis B, Einecke G, Mengel M, Chang J, et al. De novo donor-specific antibody at the time of kidney transplant biopsy associates with microvascular pathology and late graft failure. Am J Transplant. 2009 Nov;9(11):2532-41.

17 Zeevi A, Lunz J, Feingold B, Shullo M, Bermudez C, Teuteberg J, et al. Persistent strong anti-HLA antibody at high titer is complement binding and associated with increased risk of antibody-mediated rejection in heart transplant recipients. J Heart Lung Transplant. 2013 Jan;32(1):98-105.

18 Safavi S, Robinson DR, Soresi S, Carby M, Smith JD. De novo donor HLA-specific antibodies predict development of bronchiolitis obliterans syndrome after lung transplantation. J Heart Lung Transplant. 2014 Dec; 33(12):1273-81.

19 Girnita AL, Duquesnoy R, Yousem SA, Iacono AT, Corcoran TE, Buzoianu M, et al. HLAspecific antibodies are risk factors for lymphocytic bronchiolitis and chronic lung allograft dysfunction. Am J Transplant. 2005 Jan;5(1):131-8.

20 Sundaresan S, Mohanakumar T, Smith MA, Trulock EP, Lynch J, Phelan D, et al. HLA-A locus mismatches and development of antibodies to HLA after lung transplantation correlate with the development of bronchiolitis obliterans syndrome. Transplantation. 1998 Mar;65(5):648-53.

21 Roux A, Thomas KA, Sage E, SuberbielleBoissel C, Beaumont-Azuar L, Parquin F, et al. Donor-specific HLA antibody-mediated complement activation is a significant indicator of antibody-mediated rejection and poor long-term graft outcome during lung transplantation: a single center cohort study. Transpl Int. 2018 Jul;31(7):761-72.

22 Hachem RR, Yusen RD, Meyers BF, Aloush AA, Mohanakumar T, Patterson GA, et al. Anti-human leukocyte antigen antibodies and preemptive antibody-directed therapy after lung transplantation. J Heart Lung Transplant. 2010 Sep;29(9):973-80.

23 Rose EA, Barr ML, Xu H, Pepino P, Murphy MP, McGovern MA, et al. Photochemotherapy in human heart transplant recipients at high risk for fatal rejection. J Heart Lung Transplant. 1992 Jul-Aug;11(4 Pt 1):746-50.

24 Barr ML, Baker CJ, Schenkel FA, McLaughlin SN, Stouch BC, Starnes VA, et al. Prophylactic photopheresis and chronic rejection: effects on graft intimal hyperplasia in cardiac transplantation. Clin Transplant. 2000 Apr;14(2): 162-6.
25 Baskaran G, Tiriveedhi V, Ramachandran S, Aloush A, Grossman B, Hachem R, et al. Efficacy of extracorporeal photopheresis in clearance of antibodies to donor-specific and lung-specific antigens in lung transplant recipients. J Heart Lung Transplant. 2014 Sep; 33(9):950-6.

26 Maeda A, Schwarz A, Kernebeck K, Gross N, Aragane Y, Peritt D, et al. Intravenous infusion of syngeneic apoptotic cells by photopheresis induces antigen-specific regulatory T cells. J Immunol. 2005 May;174(10):596876.

27 Bladon J, Taylor P. Extracorporeal photopheresis reduces the number of mononuclear cells that produce pro-inflammatory cytokines, when tested ex-vivo. J Clin Apher. 2002;17(4):177-82.

28 Ensor CR, Yousem SA, Marrari M, Morrell MR, Mangiola M, Pilewski JM, et al. Proteasome Inhibitor Carfilzomib-Based Therapy for Antibody-Mediated Rejection of the Pulmonary Allograft: Use and Short-Term Findings. Am J Transplant. 2017 May; 17(5): 1380-8.

29 Böhmig GA, Wahrmann M, Regele H, Exner M, Robl B, Derfler K, et al. Immunoadsorption in severe C4d-positive acute kidney allograft rejection: a randomized controlled trial. Am J Transplant. 2007 Jan;7(1):117-21.

30 Palmer SM, Davis RD, Hadjiliadis D, Hertz MI, Howell DN, Ward FE, et al. Development of an antibody specific to major histocompatibility antigens detectable by flow cytometry after lung transplant is associated with bronchiolitis obliterans syndrome. Transplantation. 2002 Sep;74(6):799-804.

31 DeVos JM, Gaber AO, Knight RJ, Land GA, Suki WN, Gaber LW, et al. Donor-specific HLA-DQ antibodies may contribute to poor graft outcome after renal transplantation. Kidney Int. 2012 Sep;82(5):598-604.

32 Campos EF, Tedesco-Silva H, Machado PG, Franco M, Medina-Pestana JO, Gerbase-DeLima M. Post-transplant anti-HLA class II antibodies as risk factor for late kidney allograft failure. Am J Transplant. 2006 Oct; 6(10):2316-20.

33 Langan LL, Park LP, Hughes TL, Irish A, Luxton G, Witt CS, et al. Post-transplant HLA class II antibodies and high soluble CD30 levels are independently associated with poor kidney graft survival. Am J Transplant. 2007 Apr;7(4):847-56.

34 Reinsmoen NL, Nelson K, Zeevi A. Anti-HLA antibody analysis and crossmatching in heart and lung transplantation. Transpl Immunol. 2004 Jun-Jul;13(1):63-71.

35 Cunningham AC, Zhang JG, Moy JV, Ali S, Kirby JA. A comparison of the antigen-presenting capabilities of class II MHC-expressing human lung epithelial and endothelial cells. Immunology. 1997 Jul;91(3):458-63.

36 Ius F, Sommer W, Tudorache I, Kühn C, Avsar M, Siemeni T, et al. Preemptive treatment with therapeutic plasma exchange and rituximab for early donor-specific antibodies after lung transplantation. J Heart Lung Transplant. 2015 Jan;34(1):50-8. 